\title{
Effect of roflumilast in patients with severe COPD and a history of hospitalisation
}

To the Editor:

Exacerbations of chronic obstructive pulmonary disease (COPD) are acute events characterised by a worsening of respiratory symptoms that necessitates a change in medication and/or hospital admission [1]. Severe exacerbations (those resulting in hospitalisation and/or death) are associated with a poor prognosis [2], decreased quality of life [3] and higher mortality [2,4]. Roflumilast is a once-daily, oral, selective phosphodiesterase-4 inhibitor that targets both the systemic and pulmonary inflammation associated with COPD [5]. Previous studies have shown that roflumilast as an add-on to inhaled therapies reduces COPD exacerbations in patients with severe COPD who have chronic bronchitis and a history of exacerbations [6-9]. However, the adverse events associated with systemic therapies such as roflumilast necessitate that these therapies are used to treat specific patient populations in whom they are likely to have the greatest clinical benefit [10].

Recently, the REACT study investigated the effects of roflumilast in a well-defined patient population with severe COPD who experienced exacerbations despite treatment with inhaled corticosteroid/long-acting $\beta_{2}$-agonist \pm long-acting muscarinic antagonist (ICS/LABA \pm LAMA) [11]. The detailed methodology for REACT has been published previously [11]. In brief, it was a double-blind, placebo-controlled, multinational study that included patients with severe airflow limitation (confirmed by a post-bronchodilator forced expiratory volume in $1 \mathrm{~s}(\mathrm{FEV} 1) /$ forced vital capacity ratio $<0.70$ and a post-bronchodilator FEV $1 \leqslant 50 \%$ predicted), symptoms of chronic bronchitis and a history of at least two exacerbations in the previous year. Patients were randomised (1:1) to receive once-daily roflumilast $500 \mu \mathrm{g}$ or placebo for 52 weeks, added to ICS/LABA \pm LAMA (70\% of patients used concomitant LAMA).

The primary end-point was the rate of moderate (treated with systemic steroids) or severe (leading to hospitalisation/death) COPD exacerbations per patient per year. When analysed by Poisson regression, the frequency of moderate or severe exacerbations was $13.2 \%$ numerically lower in the roflumilast group versus placebo, but did not reach statistical significance (rate ratio (RR) $0.868,95 \%$ CI $0.753-1.002 ; \mathrm{p}=0.0529$ ), with a statistically significant $14.2 \%$ reduction versus placebo ( $R R$ $0.858,95 \%$ CI $0.740-0.995 ; p=0.0424$ ) when analysed by predefined sensitivity analysis using negative binomial regression [11].

REACT included a large number of patients who had been hospitalised with a severe COPD exacerbation in the year before randomisation [12], a population often excluded from clinical trials. We therefore undertook a post hoc analysis of REACT to explore the effect of roflumilast in patients hospitalised for a COPD exacerbation in the previous year. End-points included exacerbation rate, time to first and next severe exacerbation (i.e. rehospitalisation), change in post-bronchodilator FEV1 and change in COPD Assessment Test (CAT) score, as well as adverse events. We used negative binomial regression to estimate exacerbation rates, a Cox proportional hazards regression model to estimate time to first and next severe exacerbation, and an analysis of covariance to calculate the change in post-bronchodilator FEV1 and total CAT score. All analyses were done in the intention-to-treat (ITT) population. In this exploratory analysis, results are reported as significant at the nominal level of $5 \%$.

Of the 1935 patients recruited, 641 (33\%) had been hospitalised with a COPD exacerbation in the previous year (roflumilast $n=322$; placebo $n=319$ ), while 1294 had not (roflumilast $n=647$; placebo $n=647$ ). There were more current smokers in the subgroup with a history of hospitalisation than in the subgroup without

@ERSpublications

Roflumilast provides greater therapeutic benefit in severe COPD patients with a hospitalisation in the prior year http://ow.ly/lzWy30bNBd4

Cite this article as: Rabe KF, Calverley PMA, Martinez FJ, et al. Effect of roflumilast in patients with severe COPD and a history of hospitalisation. Eur Respir J 2017; 50: 1700158 [https://doi.org/10.1183/ 13993003.00158-2017]. 
hospitalisation (51.6\% and 39.6\%, respectively). Other demographic and disease characteristics were similar between the two subgroups, with a mean age of 64.7 years and post-bronchodilator FEV1 of $34.3 \%$ predicted in the prior hospitalisation subgroup.

Among patients with a prior hospitalisation, the rate of moderate or severe exacerbations was $22.9 \%$ lower in the roflumilast versus placebo group (RR $0.771,95 \%$ CI $0.610-0.974 ; p=0.029$ ) and the rate of severe exacerbations was $34.9 \%$ lower ( $R R$ 0.651, 95\% CI 0.477-0.887; $\mathrm{p}=0.007$ ) (figure 1). In patients without a hospitalisation for COPD exacerbation in the previous year, the reductions in the rate of moderate or severe and severe exacerbations with roflumilast were $8.6 \%$ and $7.6 \%$, respectively. Neither was significantly different compared with placebo (figure 1).

In patients hospitalised for COPD exacerbation in the previous year, roflumilast significantly prolonged time to first severe exacerbation (hazard ratio (HR) $0.708,95 \%$ CI $0.525-0.949 ; \mathrm{p}=0.0218$ ) and median time to rehospitalisation ( 143 days versus 136 days; HR $0.706,95 \%$ CI $0.523-0.948 ; \mathrm{p}=0.0214$ ) versus placebo. There was no significant difference between roflumilast and placebo in time to a first severe exacerbation in patients not hospitalised for a COPD exacerbation in the previous year (HR 0.963, 95\% CI 0.705-1.311; $\mathrm{p}=0.8096)$.

Additionally, post-bronchodilator FEV1 increased significantly with roflumilast treatment over the 52 weeks in both hospitalisation subgroups $(\Delta=47 \mathrm{~mL}$ and $\Delta=60 \mathrm{~mL}$ versus placebo in patients with and without a prior hospitalisation). Changes in total CAT score were not significantly different between roflumilast and placebo in either hospitalisation subgroup (least squares (LS) mean 0.152, $\mathrm{p}=0.7049$ in patients with a prior hospitalisation; LS mean $-0.492, \mathrm{p}=0.0562$ in patients with no prior hospitalisation).

The proportion of patients experiencing adverse events was similar across patient groups irrespective of their hospitalisation history. Among those hospitalised for a COPD exacerbation, adverse events were reported by $223(69.3 \%)$ and $220(69 \%)$ patients receiving roflumilast and placebo, respectively, and in the no prior hospitalisation subgroup by $425(65.8 \%)$ and $352(54.3 \%)$ patients, respectively. Serious adverse events were reported by 109 (33.9\%) and 137 (42.9\%) patients receiving roflumilast and placebo, respectively, within the prior hospitalisation subgroup, while $140(21.7 \%)$ and $148(22.8 \%)$ patients in the no prior hospitalisation subgroup experienced a serious adverse event. The most frequent adverse events associated with roflumilast were diarrhoea, weight loss and nausea, consistent with previous studies [7-9].

Overall, this post hoc analysis of REACT has identified a subgroup of patients that derived greater benefit from roflumilast in reducing the rate of moderate or severe exacerbations. This subgroup finding has since been corroborated in the $\mathrm{RE}^{2} \mathrm{SPOND}$ study, a similarly designed 52-week study [13]. Similar to REACT, 32\% (745/2352) of patients in $\mathrm{RE}^{2}$ SPOND had been hospitalised for a COPD exacerbation in the previous year. Addition of roflumilast to ICS/LABA \pm LAMA resulted in an $8.5 \%$ reduction in the rate of moderate or severe exacerbations per patient per year (primary end-point), which was not statistically
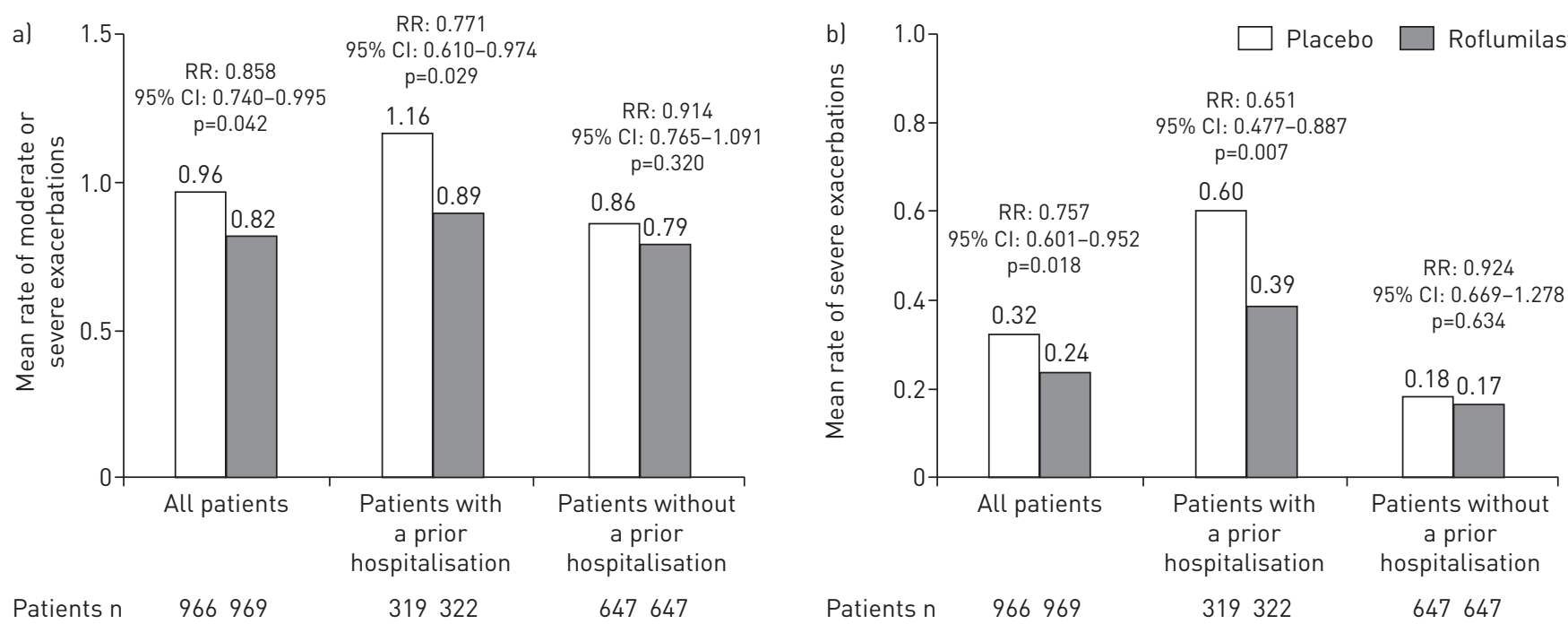

FIGURE 1 a) Rate of moderate or severe exacerbations per patient per year by history of hospitalisation in the previous year. b) Rate of severe exacerbations per patient per year by history of prior hospitalisation in the previous year. Rate ratios (RR), 95\% confidence intervals and p-values are based on a negative binomial regression analysis in the intention-to-treat population. Moderate exacerbations were defined as those requiring treatment with systemic steroids. Severe exacerbations were defined as those that led to hospital admission and/or death. 
significant ( $R R$ 0.92, 95\% CI 0.81-1.04, p=0.163). However, post hoc analyses showed that roflumilast significantly reduced the rate of moderate or severe exacerbations in two patient subgroups: those with $>3$ exacerbations (39\% versus placebo ( $\mathrm{RR} 0.61,95 \%$ CI $0.39-0.95 ; \mathrm{p}=0.030)$ ) and those with $\geqslant 1$ hospitalisation for a COPD exacerbation (25\% versus placebo (RR 0.75 , 95\% CI 0.60-0.93; $\mathrm{p}=0.010)$ ) 1 year prior to randomisation [13].

Although REACT and RE ${ }^{2}$ SPOND were generally similar in design, methodological differences between the two studies included the formulation of roflumilast used (European Medicines Agency-approved enteric film-coated tablets in REACT and US Food and Drug Administration (FDA)-approved uncoated tablets in $\mathrm{RE}^{2}$ SPOND), as well as the maximum dose of ICS/LABA allowed in each study (for fluticasone/ salmeterol, the maximum approved dose in each participating country, mostly 500/50 $\mu \mathrm{g}$ (one inhalation twice daily) was allowed in REACT and the maximum FDA-approved dose of 250/50 $\mu \mathrm{g}$ (one inhalation twice daily) in $\mathrm{RE}^{2} \mathrm{SPOND}$ ) [13].

Together, the results from REACT and $\mathrm{RE}^{2} \mathrm{SPOND}$ indicate that roflumilast is most effective at reducing the rate of moderate or severe exacerbations in a subgroup of patients with a hospitalisation for a COPD exacerbation in the previous year. Furthermore, in this COPD phenotype, roflumilast effectively reduced the rate of exacerbations that require hospitalisation, which are the most serious and costly of COPD exacerbations [14], and reduced the risk of re-admission to hospital due to another COPD exacerbation. We speculate that these COPD patients have more pronounced inflammation than those with less advanced disease and that the reduction in exacerbation risk observed with roflumilast may relate to its anti-inflammatory properties. The benefit of reducing the risk of rehospitalisation in the most severe COPD patients is of major significance to patients and payers. These results help to further define the specific COPD patient phenotype that derives the most benefit from roflumilast therapy.

Klaus F. Rabe ${ }^{1,5}$, Peter M.A. Calverley ${ }^{2,5}$, Fernando J. Martinez ${ }^{3}$ and Leonardo M. Fabbri $\oplus^{4}$

${ }^{1}$ LungenClinic Grosshansdorf, Airway Research Center North, Member of the German Center for Lung Research (DZL), Grosshansdorf, Germany. ${ }^{2}$ Institute of Ageing and Chronic Disease, University of Liverpool, Liverpool, UK. ${ }^{3}$ Weill Cornell Medical College, New York, NY, USA. ${ }^{4}$ University of Modena and Reggio Emilia, Modena, Italy. ${ }^{5}$ Co-primary authors.

Correspondence: Klaus F. Rabe, LungenClinic Grosshansdorf, Airway Research Center North, Member of the German Center for Lung Research (DZL), Wohren Damm 8022927, Grosshansdorf, Germany. E-mail: kfrabe@lungenclinic.de

Received: Jan 232017 | Accepted after revision: March 292017

Clinical trial: This study is registered with clinicaltrials.gov with the identifier NCT01329029.

Support statement: This study was funded by Takeda Pharmaceutical Company and AstraZeneca. Funding information for this article has been deposited with the Crossref Funder Registry.

Conflict of interest: Disclosures can be found alongside this article at erj.ersjournals.com

Acknowledgements: We thank Alexis Pashiardis (Synergy Vision, London, UK; supported by AstraZeneca) for writing and editorial assistance with the preparation of this research letter. These results were presented at the European Respiratory Society International Congress in 2015.

\section{References}

1 Global Initiative for Chronic Obstructive Pulmonary Disease (GOLD) 2017. www.goldcopd.org Date last accessed: December 2016.

2 Müllerova H, Maselli DJ, Locantore N, et al. Hospitalized exacerbations of COPD: risk factors and outcomes in the ECLIPSE cohort. Chest 2015; 147: 999-1007.

3 Seemungal TA, Donaldson GC, Paul EA, et al. Effect of exacerbation on quality of life in patients with chronic obstructive pulmonary disease. Am J Respir Crit Care Med 1998; 157: 1418-1422.

4 Soler-Cataluña JJ, Martínez-García MA, Román Sánchez P, et al. Severe acute exacerbations and mortality in patients with chronic obstructive pulmonary disease. Thorax 2005; 60: 925-931.

5 Wedzicha JA, Calverley PM, Rabe KF. Roflumilast: a review of its use in the treatment of COPD. Int J COPD 2016; 11: 81-90.

6 Bateman ED, Rabe KF, Calverley PM, et al. Roflumilast with long-acting $\beta_{2}$-agonists for COPD: influence of exacerbation history. Eur Respir J 2011; 38: 553-560.

7 Rennard SI, Calverley PM, Goehring UM, et al. Reduction of exacerbations by the PDE4 inhibitor roflumilast the importance of defining different subsets of patients with COPD. Respir Res 2011; 12: 18.

8 Fabbri LM, Calverley PM, Izquierdo-Alonso JL, et al. Roflumilast in moderate-to-severe chronic obstructive pulmonary disease treated with longacting bronchodilators: two randomised clinical trials. Lancet 2009; 374: 695-703.

9 Calverley PM, Rabe KF, Goehring UM, et al. Roflumilast in symptomatic chronic obstructive pulmonary disease: two randomised clinical trials. Lancet 2009; 374: 685-694.

10 Agusti A. The path to personalised medicine in COPD. Thorax 2014; 69: 857-864. 
11 Martinez FJ, Calverley PM, Goehring UM, et al. Effect of roflumilast on exacerbations in patients with severe chronic obstructive pulmonary disease uncontrolled by combination therapy (REACT): a multicentre randomised controlled trial. Lancet 2015; 385: 857-866.

12 Martinez FJ, Calverley PM, Goehring U-M, et al. Effect of roflumilast on exacerbations in patients with severe COPD and a prior history of hospitalization taking combination therapy. Eur Respir J 2015; 46: Suppl. 59, OA482.

13 Martinez FJ, Rabe KF, Sethi S, et al. Effect of roflumilast and inhaled corticosteroid/long-acting $\beta_{2}$-agonist on chronic obstructive pulmonary disease exacerbations ( $\mathrm{RE}^{2} \mathrm{SPOND}$ ). A randomized clinical trial. Am J Respir Crit Care Med 2016; 194: 559-567.

14 López-Campos JL, Tan W, Soriano JB. Global burden of COPD. Respirology 2016; 21: 14-23.

Copyright @ERS 2017 